Journal of Computer Science 4 (10): 857-863, 2008

ISSN 1549-3636

(C) 2008 Science Publications

\title{
Separating Low Pass and High Pass Frequencies in the Image Without loosing information
}

\author{
Ziad Alqadi, Musbah J. and Aqel Luis Montengro \\ University Avenue and West 181 Street, Bronx, 10453, City University of New York
}

\begin{abstract}
Granulometries are morphological image analysis tools that are particularly useful for estimating object sizes in binary and grayscale images, or for characterizing textures based on their pattern spectra. There are many applications that morphology can be applied to. Some morphological operations like dilation, erosion and open-close are used in image and video processing, but with no application to pre-processing for increasing a Codec's performance. These operations can be used as filters and if applied on an image, some information will be lost. In order to avoid information loose a sequence of morphological operation is suggested, this sequence can be used to separate low pass and high pass frequencies from the image without throwing any piece of data and the original image can always be reconstructed.
\end{abstract}

Key words: Dilation, erosion, opening, closing, gray-level morphology, granulometry

\section{INTRODUCTION}

Mathematical Morphology is the analysis of signals in terms of shape. This simply means that morphology works by changing the shape of objects contained within the signal. Mathematical morphology was developed in the 1970's by Matheron ${ }^{[1]}$ and Serra $^{[2]}$. Morphology has several advantages over other techniques especially when applied to image processing.

Some morphological operations like dilation, erosion and open-close are used in image and video processing. For example, dilation extends the boundary of object by removing low valued regions. Erosion contracts the boundaries by removing high valued regions. Distortions can bee seen in some of these images. The distortion generated not only depends on the filter but also on the image.

Opening will remove high intensity points whilst keeping the rest of the image intact. Closing is doing the opposite of this, removing low valued points whilst keeping the rest of the image intact. Both Open-Close and Close-Open remove both high and low valued points while keeping the rest of the image intact. However, the last two operations do not give the same result due to the order of the erosions and dilations.

The above mentioned operations can be used as filters and applying these filters on an image can results in loose of some information.

In order to avoid information loose a sequence of morphological operation is suggested, this sequence can be used to separate low pass and high pass frequencies from the image without throwing any piece of data and the original image can always be reconstructed.

There are many more applications that morphology can be applied to. Morphology has been widely researched for use in image and video processing, but with no application to pre-processing for increasing a Codec's performance.

Morphological operations: Morphology uses Set Theory as the foundation for many functions ${ }^{[8]}$. The simplest functions to implement are 'Dilation' and 'Erosion'. Dilation extends the boundary of object by removing low valued regions. Erosion contracts the boundaries by removing high valued regions. Distortions can be seen in some of these images. The distortion generated not only depends on the filter but also on the image.

Opening will remove high intensity points whilst keeping the rest of the image intact. Closing is doing the opposite of this, removing low valued points whilst keeping the rest of the image intact. Both Open-Close and Close-Open remove both high and low valued points while keeping the rest of the image intact. However, the last two operations do not give the same result due to the order of the erosions and dilations.

Dilation in 1D is defined as:

$$
\mathrm{A} \oplus \mathrm{B}=\{\mathrm{x}:(\hat{\mathrm{B}}) \mathrm{x} \cap \mathrm{A} \neq \varnothing\}=\bigcup_{\mathrm{x} \in \mathrm{B}} \mathrm{A}_{\mathrm{x}}
$$

Corresponding Author: Ziad Alqadi, University Avenue and West 181 street, Bronx, NY 10453. City University of New York 
where, A and B are sets in Z. This definition is also known as Minkowski Addition ${ }^{[6-9]}$. This equation simply means that $\mathrm{B}$ is moved over $\mathrm{A}$ and the intersection of $\mathrm{B}$ reflected and translated with $\mathrm{A}$ is found. Usually A will be the signal or image being operated on and B will be the Structuring Element. Equation 1 is used to process binary sets of data. Dilation has several interesting properties, which make it useful for image processing. These properties can be very useful in image processing and can result in some operations being simplified.

Erosion: The opposite of dilation is known as erosion. This is defined as:

$$
\mathrm{A} \square=\{\mathrm{x}:\{\mathrm{B}\} \mathrm{x} \subseteq \mathrm{A}\}=\bigcap_{\mathrm{x} \subset \mathrm{B}} \mathrm{Ax}
$$

This definition is also known as Minkowski Subtraction $^{[6-8]}$. The equation simply says, erosion of $\mathrm{A}$ by $B$ is the set of points $\mathrm{x}$ such that $\mathrm{B}$ translated by $\mathrm{x}$ is contained in A. Figure 2 shows how erosion works on a 1D binary signal. This works in exactly the same way as dilation. However (6) essentially says that for the output to be a one, all of the inputs must be the same as the structuring element. Thus, erosion will remove runs of ones that are shorter than the structuring element. Erosion, like dilation also contains properties that are useful for image processing:

Opening and closing: Both dilation and erosion have interesting and useful properties. However, it would be useful to have the properties of both in one function. This can be done in two ways. The first method, 'Opening', is defined as ${ }^{[5,8]}$ :

$$
\mathrm{A} \square \mathrm{B}=\{\mathrm{A} \square \mathrm{B}\} \oplus \mathrm{B}
$$

This simply erodes the signal and then dilates the result as shown in Fig. 1. As can be seen, the zeros are opened up. Any ones that are shorter than the structuring element are removed, but the rest of the signal is left unchanged. This is a very useful property as it means that if the filter is applied once, no more changes to the signal will result from repeated applications is known as 'Idempotent' ${ }^{\text {[8]. }}$

$$
\{\mathrm{AOB}\} \mathrm{OB}=\mathrm{AOB}
$$

The opposite of opening, is 'Closing' defined by:

$$
\mathrm{A} \bullet \mathrm{B}=(\mathrm{A} \oplus \mathrm{B}) \square \mathrm{B}
$$
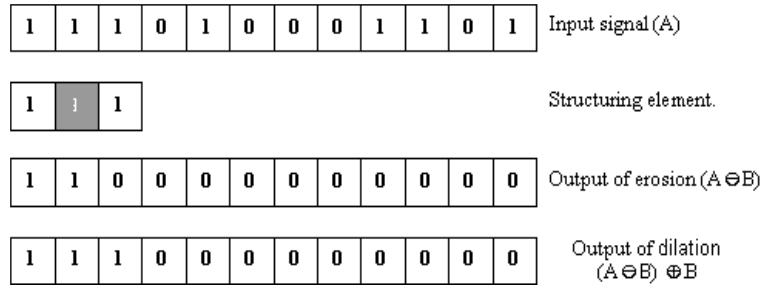

Fig. 1: Example of how an opening works

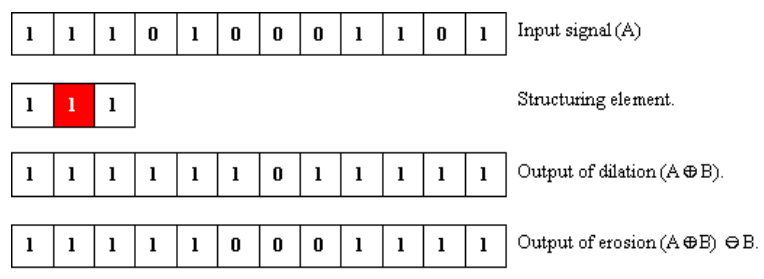

Fig. 2: Example of how a closing works

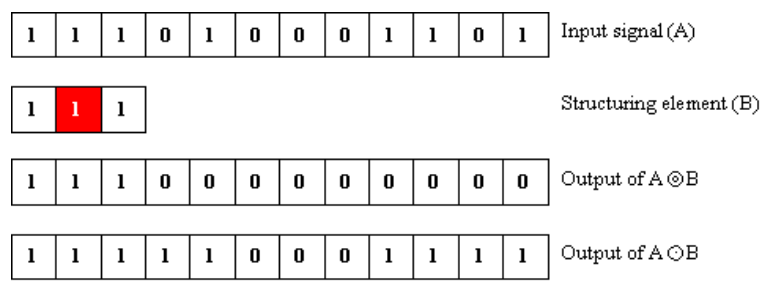

Fig. 3: Example of how open close and close open work

Figure 2 shows how this study. It can be seen that this closes gaps in the signal in the same way as opening opened up gaps. Closing also has the property of being idempotent.

Both of these filters again have interesting properties that would be nice to have in one filter. The opening and closing can be combined to merge these properties (Fig. 3). There are two ways of combining these, the first of which is known as an 'Open-Close' filter and is defined by:

$$
\mathrm{A} \odot \mathrm{B}=\{\mathrm{AOB}\} \bullet \mathrm{B}
$$

The signal is first opened and the result is then closed. The opposite can also be done by closing and then opening. This is called a 'Close-Open' filter and is defined by:

$$
\mathrm{A} \square \mathrm{B}=\{\mathrm{A} \bullet \mathrm{B}\} \mathrm{OB}
$$

Extending to grey scale: For morphology to be of use in image processing, it needs to be extended to non- 
binary signals. There are various ways in which this can be done ${ }^{[3]}$. The chosen method uses very simple functions, which allow them to be implemented in an efficient way ${ }^{[11]}$. The following sections describe one method of implementing grey scale morphology.

Grey scale dilation: When applying dilation it should be clear that this is actually taking the maximum value lying within the structuring element. Hence, dilation can be redefined for grey scale as:

$$
\mathrm{A} \oplus \mathrm{B}=\max _{\mathrm{i} \in \mathrm{B}, \forall \mathrm{x}}\left(\mathrm{A}_{\mathrm{k}+\mathrm{i}}\right)
$$

This study in exactly the same way as before, but just takes the maximum value laying within the 1's of the structuring element as shown in Fig. 4. This method of using the values where the structuring element is 1 , is known as a Flat Structuring Element (FSE). There is another method ${ }^{[5]}$ that is used in grayscale image processing.

As well as having the structuring element to choose the input elements to use, another set is used to allow a value to be added to the values used. This is sometimes written as one set as shown in Fig. 5:

$$
\mathrm{A} \oplus \mathrm{B}=\max _{\mathrm{i} \subset \mathrm{B}, \forall \mathrm{x}}\left\{\mathrm{A}_{\mathrm{k}+\mathrm{i}}+\mathrm{B}_{\mathrm{i}}\right\}
$$

Grey scale Erosion: Like dilation, from (6) it should be clear that all erosion is actually doing is taking the minimum value from within the structuring element. Thus, erosion can be redefined as:

$$
\mathrm{A} \square \mathrm{B}=\min _{\mathrm{i} \in \mathrm{B}, \forall \mathrm{x}}\left\{\mathrm{A}_{\mathrm{k}+\mathrm{i}}\right\}
$$

\begin{tabular}{|c|c|c|c|c|c|c|c|c|c|c|c|c|}
\hline 1 & 7 & 2 & 3 & $\mathbf{0}$ & 3 & 6 & 5 & 2 & 2 & 1 & 9 & Input signal $(A)$. \\
\hline 1 & 1 & 1 & & & & & & & & & & Structuring element $(B)$. \\
\hline 7 & 7 & 7 & 3 & 3 & 6 & 6 & 6 & 5 & 2 & 9 & 9 & Output of grey scale dilation. \\
\hline
\end{tabular}

Fig. 4: Example of how dilation works

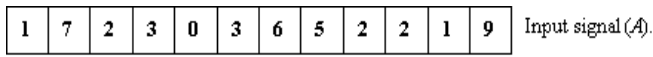

\section{\begin{tabular}{l|l|l|}
\hline 1 & 1 & 1 \\
\hline
\end{tabular}$\quad$ Structuring element $(B)$}

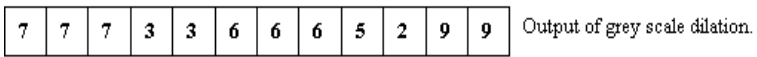

Fig. 5: Example of how a variation on standard dilation works
Both of these can still be applied to binary signals, but more importantly, they can be applied to real numbers. The rest of the functions, opening and closing and so on remain unchanged, but use the above definition for grey scale.

Extending to two dimensions: Now to be able to use morphology in image processing, the definitions need to be applied in two Dimensions (2D). This can be done relatively easily as described below ${ }^{[12-14]}$.

2D Dilation: The signal has now become a 2D signal, now called an image and hence the structuring element is changed to become $2 \mathrm{D}$, where the first structuring element is known as the Four Nearest Neighbors $(4 \mathrm{nn})$. This limits the operations to work only on horizontal and vertical components. To overcome this problem and allow diagonal components to also be used, the Eight Nearest Neighbors $(8 \mathrm{nn})$ can be used. Dilation can now be redefined as:

$$
\mathrm{A} \oplus \mathrm{B}=\max _{\{\mathrm{i}, \mathrm{j}\} \subset \mathrm{B}}\left\{\mathrm{A}_{\mathrm{x}+\mathrm{i}, \mathrm{y+j}+\mathrm{j}}\right\}
$$

This still works in the same way as before. The structuring element is moved across the image as before. The maximum value inside the structuring element is then set as the output.

2D Erosion: Erosion can also be redefined in exactly the same way, as dilation was to give a new $2 \mathrm{D}$ erosion definition:

$$
\mathrm{A} \square \mathrm{B}=\min _{\{\mathrm{i}, \mathrm{j}\} \subset \mathrm{B}}\left\{\mathrm{A}_{\mathrm{x}+\mathrm{i}, \mathrm{y+j}+\mathrm{j}}\right\}
$$

This study in the same way as dilation with the exception of erosion takes the minimum instead of the maximum. Again, the other definitions of opening, closing, etc. remain unchanged. To use them in $2 \mathrm{D}$, they must use the above methods for erosion and dilation.

Extension to three dimensions: For video processing, it is beneficial to filter spatio-temporally (i.e., in Three Dimensions, 3D). This is done because noise is uncorrelated which means that if a pixel in the current frame is noise, then there is a strong chance that the pixel will be of a different value in other frames ${ }^{[13]}$. Hence, by using the other frames to process the current frame, the noise can be filtered out. 
3D Dilation: As before, dilation can be easily extended to process in $3 \mathrm{D}$ as defined by:

$$
\mathrm{A} \oplus \mathrm{B}=\max _{\{\mathrm{ijk}\} \subset \mathrm{B}}\left\{\mathrm{A}_{\mathrm{x}+\mathrm{iy} \mathrm{jz+k}}\right\}
$$

Now the image is 3D (i.e., a sequence) and the structuring element is now 3D. This is applied in the same way as before. The structuring element is placed on the first pixel of the first frame. The output is set to the maximum value lying within the structuring element. The structuring element is then moved across the current frame until the entire frame has been processed. Then the next frame is done in exactly the same way. The process is repeated until all the frames have been processed.

Erosion: Again, erosion can be redefined in the same way to give:

$$
\mathrm{A} \square \mathrm{B}=\min _{\{\mathrm{ij}, \mathrm{k}\} \subset \mathrm{B}}\left\{\mathrm{A}_{\mathrm{x}+\mathrm{i}, \mathrm{y}+\mathrm{j}, \mathrm{z}+\mathrm{k}}\right\}
$$

The other filters, open, close, etc. are still the same as before, but just use the above definitions for 3D.

$3 \mathrm{D}$ processing can remove more noise and smooth the images more because of the correlation between frames. This is good for compression as high frequency information is removed.

The proposed sequence of morphological operations: The concept of granulometries, introduced in the late sixties by Matheron ${ }^{[15]}$, provides a consistent framework for analyzing object and structure sizes in images.

A granulometry can simply be defined as a decreasing family of openings ${ }^{[2]}$.

Though this definition was originally meant in the context of binary image processing, it directly extends to grayscale. Moreover, granulometries by closings can also be defined as families of increasing closings.

The filters described so far have interesting properties. However, one of the properties not mentioned so far is that of 'Granules'. A granule is an element that falls through a 'Sieve' in the same way that small stones fall through a sieve. If a set of structuring elements is used with increasing size, $\{B\{r\}: r>O\}$ with the property that $B(t)$ is $B(r)$ opened for $\mathrm{t} \geq \mathrm{r}$. This just means that each structuring element is bigger than the previous. Using this, an opening can be defined to use these structuring elements:

$$
\alpha_{y}\{X\}=X O B\{r\}
$$

If this satisfies the 'semi-group' property (24), then the openings are called a 'Granulometry'. If all $\alpha_{\mathrm{r}}$ are translation invariant (7) and scale compatible (10), then it is called 'Minkowski Granulometry':

$$
\alpha_{\gamma} \alpha_{t}=\alpha_{t} \alpha_{\gamma}=\alpha_{t} t \geq r
$$

$1 D$ Grey scale granulometry: It is difficult to see from the above definition, exactly what Granulometry does. As stated above, a set of increasing size structuring elements are used. The opening used above is good, but a more useful method would be to use and Open-Close, which will remove both dark and light spots. Thus, using the previous definitions, an Open-Close can be redefined for a sieve as:

$$
\mathrm{S}_{\mathrm{m}}\{\mathrm{A}, \mathrm{B}\}=\left\{\mathrm{A} \circ \mathrm{B}_{\mathrm{m}}\right\} \bullet \mathrm{B}_{\mathrm{m}}
$$

where, $\mathrm{m}$ is the given size to use. From this it should be clear that the size will determine the data that is removed. For example, if $\mathrm{r}=2$, the corresponding structuring element will remove runs of length 2 . However, for a true sieve, this must be done in order. For example, for a target size of 4 , the input would first be processed to a size of 1 . The result would then be processed to 2 and so on until the target is reached. This is defined as:

$$
\mathrm{R}_{\mathrm{t}}\{\mathrm{A}\}=\mathrm{S}_{\mathrm{t}}\left\{\mathrm{S}_{\mathrm{t}-1}\left\{\cdots\left\{\mathrm{S}_{1}\left\{\mathrm{~A}, \mathrm{~B}_{1}\right\} \cdots\right\}, \mathrm{B}_{\mathrm{t}-1}\right\}, \mathrm{B}_{\mathrm{t}}\right\}
$$

where, $\mathrm{t}$ is the target size. The granules of the system are simply the result subtracted from the input as given by:

$$
\mathrm{G}_{\mathrm{m}}\{\mathrm{A}\}=\mathrm{S}_{\mathrm{m}}\{\mathrm{A}\}-\mathrm{S}_{\mathrm{m}-1}\{\mathrm{~A}\}
$$

Here is an example of how to get law pass frequencies $\left(S_{1}, S_{2}, S_{3}\right)$ and high pass frequencies $\left(G_{1}\right.$, $\mathrm{G}_{2}, \mathrm{G}_{3}$ ) from the original signal $\mathrm{S} 0$ using mat lab.

Let the input (original signal) S0 be: 77013330000

Start with structuring element se $1=111$.

Find S1 and G1: $S_{1}=$ imclose (imopen (S0, se1), se1), this can be done by performing the following sequence 
of morphological operations: Erosion, Dilation, Dilation and Erosion using the same structuring element:

Erosion: 70001300000

Dilation: 77013330000

Dilation: 77733333000

Erosion: (S1 = 77333330000

$\mathrm{G} 1=\mathrm{S} 0-\mathrm{S} 1=00320000000$

Find S2 and G2 using se2= 11111

S2 = imclose (imopen (S1, se2), se2) 3333333000 0

$\mathrm{G} 2=\mathrm{S} 1-\mathrm{S} 244000000000000$

Find S3 and G3 using se3 = 11111

S3 = imclose (imopen (S2, se $),$ se 3$)=333333300$ 00

$\mathrm{G} 3=\mathrm{S} 2-\mathrm{S} 3=000000000000$

It should be clear to see that the outputs on $S_{m}$, are the low-pass filtered signal whilst the outputs of $G_{m}$, are the high-passed signal. Provided no data is thrown away, the original signal can be reconstructed exactly.

2D Grey scale granulometry: The previous techniques have proven that morphology is a good for filtering. However, some images show a more noticeable degradation than others. This is due to the fact that the filters described so far all operate on the entire image with no knowledge of the image statistics. This is especially noticeable when sequences with a lot of motion are used. This section describes a filter that will act on the entire image, but that only changes local minimum and maximum points.

The method used is known as 'Area Morphology'. This is an extension of 1D grey scale granulometry. For a detailed analysis and theory behind area morphology, the reader is directed to the definition for a grey-scale Area-Opening is given as:

$$
\underset{\lambda}{\gamma}(\mathrm{X})=\underset{\mathrm{S} \subset \mathrm{A} \lambda}{v}(\mathrm{X})=\underset{\mathrm{S} \subset \mathrm{A} \lambda}{v}(\mathrm{~S} \circ \mathrm{X})
$$

where, $A_{\lambda}$ is the set of subsets which are connected and whose area is greater or equal to $\lambda$. This essentially looks at all possible openings that give a certain areasize and uses the maximum found as the result. The opposite can also be applied, that is to perform an AreaClosing:

$$
\underset{\lambda}{\alpha}(X)=\underset{S \subset A \lambda}{\wedge} \varphi_{s}(X)=\underset{S \subset A \lambda}{\wedge}(S \bullet X)
$$

Area sieves: Area morphology can be used to create 2D and 3D sieves. The basic sieve structure is used, except that an Area-OpenClose is used. An AreaOpenClose is just an Area-Open followed by an AreaClose (Fig. 6). Figure 7 shows an example of a 2D sieve. This clearly shows how a sieve works and how the granules fall through the sieve.

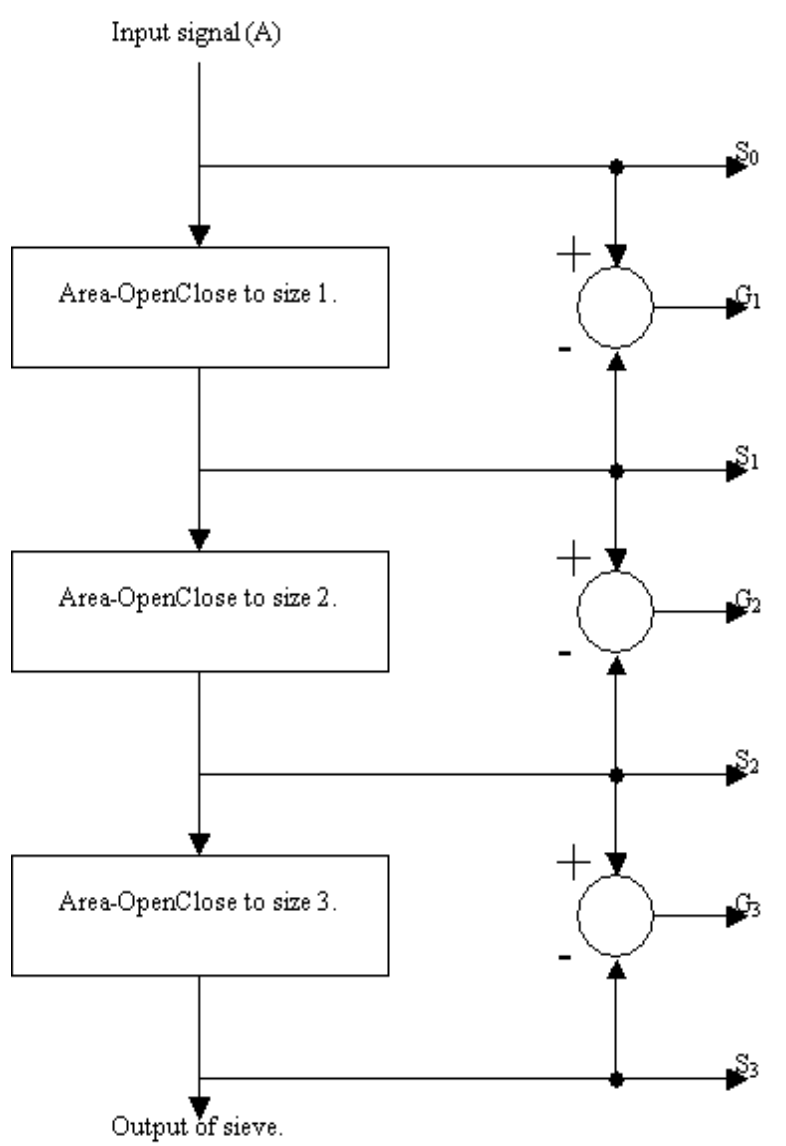

Fig. 6: Example of a 2D sieve structure using area morphology 

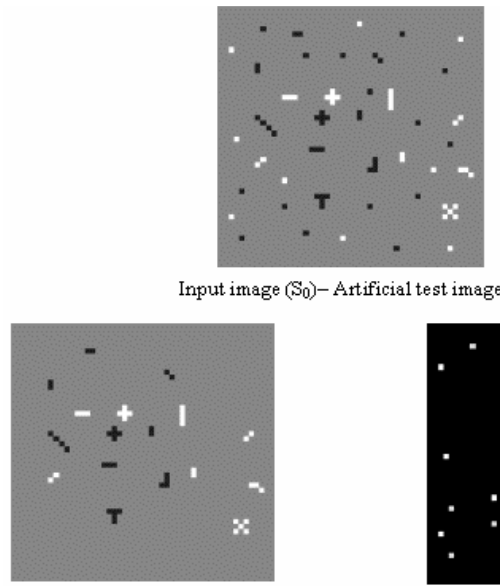

Low-pass filtered $\left(\mathrm{S}_{1}\right)$

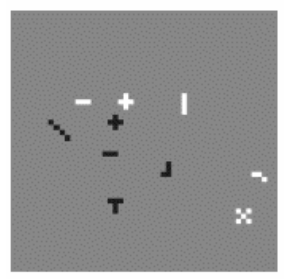

Low-pass filtered $\left(\mathrm{S}_{2}\right)$

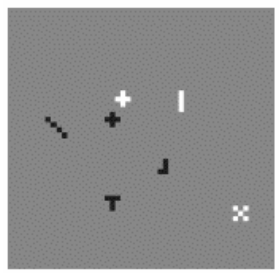

Low-pass filtered $\left(\mathrm{S}_{3}\right)$

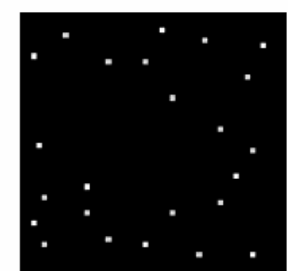

High-pass filtered - Granules of size $1\left(\mathrm{G}_{1}\right)$

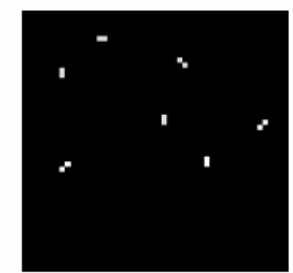

High-pass filtered - Granules of size $2\left(\mathrm{G}_{2}\right)$

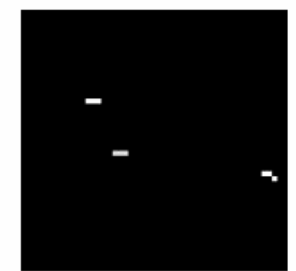

High-pass filtered - Granules of size $3\left(\mathrm{G}_{3}\right)$

Fig. 7: Example of how a sieve works on images

\section{CONCLUSION}

A sequences of morphological operations using a structuring element with increasing size was discussed and analyzed, this sequence of operations can separate the image into low pass (Sieve) and high pass (Granules) frequencies without losing any piece of information, making the reconstruction of the original image very easy. This method can again be applied to images and sequences as shown above and will cope better with motion in sequences, as the growing will actually track objects. It should also be clear that this method could also be described as a sieve. Again provided that no data is thrown away, then the original image can always be reconstructed. However, the idea is to remove parts of the image that the viewer will not notice. Hence there is little point in keeping any data other than the filtered image.

\section{REFERENCES}

1. Matheron, F., 1975. Random Sets and Integral Geometry. John Wiley and Sons, pp: 288. ISBN10: 0471576212.

2. Serra, J., 1983. 'Image Analysis and Mathematical Morphology. Academic Press, pp: 810. ISBN-10: 0126372403.

3. Bangham, J.A. and S. Marshall, 1998. Image and signal processing with mathematical morphology. Electronics commun. Eng. J., 10: 117-128. DOI: 10.1049/ecej:19980305.

4. Harvey, N.R. and S.Marshall, 1999. Restoration of archive film material using multi-dimensional soft morphological filters. Proceedings of the IEEEEURASIP Workshop on Nonlinear Signal and Image Processing, Bogazici University, Istanbul, pp: 811-815.

http://www.ee.bilkent.edu.tr/ signal/Nsip99/papers /174.pdf.

5. Gonzalez, R.C. and R.E. Woods, 2002. Digital Image Processing. 2nd Edn., Prentice Hall, pp: 793. ISBN-10: 0201600781.

6. Banon, G.J.F., 1995. Characterization of translation-invariant elementary operators for graylevel morphology. Deterministic Morphological Image Anal., 2568: 68-79. DOI: 10.1117/12.216367.

7. Braga Neto, U.M. and R.A. Lotufo, 1995. Image analysis of porous media by 3D mathematical morphology. Proceeding of the Neural, Morphological, and Stochastic Methods in Image and Signal Processing'95, São Carlos, SP-Brasil, pp: 139-150.

http://adsabs.harvard.edu/abs/1995SPIE.2568..139 B.

8. Dougherty, E.R., 1992. Mathematical Morphology in Image Processing. Illustrated Edn., CRC Press, New York, pp: 530. ISBN: 0824787242.

9. Heijmans, H.J.A.M., 1994. Morphological Image Operators. Academic Press, Inc., San Diego.

10. Humphreys, R.M. and R.L. Pennington, 1989. Workshop on digitized optical sky surveys. J. Brazilian Comput. Soc., Math. Morphol. Appro., 3.

11. Maragos, P., 1989. Pattern spectrum and multiscale shape representation. IEEE Trans. Patt. Anal. Mach. Intel., 11: 701-716. DOI: 10.1109/34.192465.

12. Serra, J., 1988. Image Analysis and Mathematical Morphology: Theoretical Advances. In: Introduction to Morphological Filters, Serra, J., (Ed.). Academic Press, Inc., London. 
13. Serra-Ricart, M., X. Calbert, L. Garrido and V. Gaitan, 1993. Multidimensional statistical analysis using artificial neural networks: Astronomical applications. Astronomical J., 104: 1685-1695.

http://adsabs.harvard.edu/abs/1993AJ....106.1685S.

14. Sternberg, S.R., 1986. Grayscale morphology. Comput. Vision, Graphics Image Process., 35: 333355. DOI: 10.1016/0734-189X(86)90004-6.
15. Mattioli, J. and M. Schmitt, 1992. On information contained in the erosion curve. NATO Shape in Picture Workshop, Sep. 7-11, Driebergen, The Netherlands, pp: 177. 